\title{
Relationship between serum irisin, glycemic indices, and renal function in type 2 diabetic patients
}

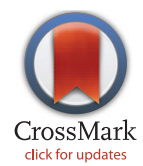

\author{
Leila Mahmoodnia ${ }^{1}$, Maryam Sadoughi ${ }^{1}$, Ali Ahmadi ${ }^{2}$, Marzieh Kafeshani ${ }^{3^{*}}$ \\ ${ }^{1}$ Department of Internal Medicine, Shahrekord University of Medical Sciences, Shahrekord, Iran \\ ${ }^{2}$ Department of Epidemiology and Biostatistics, Shahrekord University of Medical Sciences, Shahrekord, Iran \\ ${ }^{3}$ School of Nutrition \& Food Science, Isfahan University of Medical Sciences, Isfahan, Iran
}

\section{A R T I C L E IN F O}

Article Type:

Original

\section{Article History:}

Received: 23 August 2016

Accepted: 28 October 2016

Published online: 20 November 2016

\section{Keywords:}

Irisin

Type 2 diabetic patients

Body mass index

\begin{abstract}
A B S T R A C T
Introduction: Irisin is a novel peptide that plays notable role in human and animal biology and physiology. It has been reported that irisin may improve insulin resistance and related disturbances.

Objectives: The aim of this investigation was to assess the relationship between serum irisin, glycemic indices, and renal function in diabetic subjects.

Patients and Methods: In this cross-sectional study, a total of 102 type 2 diabetes mellitus (T2DM) patients were recruited. Blood biochemical parameters, including fasting plasma sugar (FBS), glycosylated hemoglobin $\left(\mathrm{HbA}_{1 \mathrm{C}}\right)$, serum uric acid (sUA), creatinine concentration and glomerular filtration rate (GFR) were measured. All statistical analysis was performed with SPSS 16.0.

Results: There was a positive correlation between irisin and age $(P=0.05, \mathrm{r}=0.19)$ and a negative correlation between irisin and body mass index (BMI) $(P=0.01, \mathrm{r}=-0.25)$ was detected. There was a significant difference of serum irisin level between patients with normal and abnormal FBS too.

Conclusion: In this study we found, irisin concentration was increased with age, decreased with BMI, and it was higher in subject with abnormal FBS. Thus further research is needed to provide inclusive understanding of irisin associated physiological effects and possible implications in clinical conditions.
\end{abstract}

Implication for health policy/practice/research/medical education:

In a study on 102 type 2 diabetic patients, we found, irisin concentration was increased with age, decreased with BMI, and it was higher in subject with abnormal FBS.

Please cite this paper as: Mahmoodnia L, Sadoughi M, Ahmadi A, Kafeshani M. Relationship between serum irisin, glycemic indices, and renal function in type 2 diabetic patients. J Renal Inj Prev. 2017;6(2):88-92. DOI: 10.15171/jrip.2017.17.

\section{Introduction}

The worldwide prevalence of diabetes was estimated 382 million people by the International Diabetes Federation in 2013. Diabetes mellitus is a complex public health concern that is related with various micro-vascular (neuropathy, nephropathy, and retinopathy) and macro-vascular (peripheral arterial disease, coronary artery disease, and stroke) complications. These complications cause vast disability, morbidity, and mortality, and even low quality of life. There are different factors that influence on the risk of developing diabetes and its complication (1).

Recently, researchers have shown an increased interest in association between irisin level and diseases. Irisin is a novel peptide that plays notable role in human and animal biology and physiology. It has been reported that irisin may improve body weight states and insulin resistance. Irisin was first introduced as exercise-induced myokines by Boström et al in 2012, but other studies showed it also is an adipokine that secreted by subcutaneous adipocytes and affected by exercise and nutritional factors (2). In brief, Boström et al demonstrated that exercise enhances levels of peroxisome proliferator-activated receptor- $\gamma$ coactivator-1 $\alpha$ (PGC-1 $\alpha$ ) in the muscle thus the expression of the type I membrane precursor protein fibronectin-type 
III domain-containing 5 (FNDC5) was induced. Then irisin was produced by attaching $\mathrm{N}$-terminal fibronectin III (FNIII)-like domain to a flexible C-terminal. Irisin stimulated the expression of brown adipose tissue (BAT) related genes such as uncoupling protein 1 (UCP1, highly expressed in BAT and a marker of browning), partially through increased PPAR- $\alpha$ and other incompletely understood method (3). Lesser circulating irisin was related with the insulin resistance, diabetes $(4,5)$, chronic kidney disease $(6,7)$ and other disease (8). The results of studies on irisin and glucose homeostasis have been controversial. Some studies established that serum irisin was significantly negatively correlated with hemoglobin $\mathrm{A}_{1 \mathrm{C}}$, fasting blood glucose, 2 hours plasma glucose and homeostasis model assessment of insulin resistance (HOMA-IR) and other studies found positive correlation (9). Studies regarding relationship between serum irisin and renal functional markers are scares however these researches suggested that irisin level might be associated with renal function in humans.

\section{Objectives}

According to the limited studies and the controversy about scientific evidence, there is a need for further research to identify the relationship between irisin, and clinical and biochemical markers of various chronic diseases. The main aim of this investigation was to assess the relationship between serum irisin, glycemic indices, and renal function in diabetic individuals.

\section{Patients and Methods}

\section{Patients}

In this cross-sectional study, a total of 102 type 2 diabetes mellitus (T2DM) patients were recruited from ImamAli clinic, Shahrekord, Iran. In the present study, T2DM was defined using the American Diabetes Association (ADA) criteria. Standard questionnaire was used for assessing information, including age, sex, disease history, anti-diabetic and anti-hypertensive drugs. Diabetic patients on dialysis, patients with kidney transplants, and mentally retarded patients who were not able to give informed consent were excluded. Additionally patients with malignancy and active or chronic infections were excluded.

\section{Ethical issues}

The research adhered to the principles of the Declaration of Helsinki; the purpose and design of study were explained for all subjects and they completed an informed consent form. Ethical Committee of Shahrekord University of Medical Science approved the protocol (\#IR.SKUMS.REC 1394.165).

\section{Biochemical assessments}

Blood biochemical parameters, including fasting plasma sugar (FBS), serum uric acid (sUA) and creatinine (Cr) concentration were measured by commercially available enzyme assay kits (Pars Azmon kit, Iran). HbA $1 \mathrm{C}$ was measured with chromatography using Biosystem kit (Spain). Glomerular filtration rate (GFR) was estimated from the results of blood Cr test and by the Modification of Diet in Renal Disease (MDRD) equation. Circulating irisin was quantified using a commercial ELISA kit (BioVendor - Laboratorni Medicina, Czech Republic).

\section{Clinical measurements}

Blood pressure (BP) was determined in a seated position where their arm sustained at heart level, after 5 minute rest. $\mathrm{BP}$ was noted as three sequential amounts at intervals of 30 seconds. The mean of the three BP measurements was utilized in the analysis.

\section{Statistical analysis}

The normality of data was tested by using KolmogorovSmirnov test. The nonparametric statistical methods were used to analyses the numerical data because the data had no normal distribution. Parametric variables were extracted as mean \pm standard deviation (SD). Categorical data were stated as percent and compared by $\chi^{2}$ test. Median and quartile were used to describe nonparametric data. Mann-Whitney U rank test was used to compare differences between groups. Kruskal-Wallis one-way analysis of variance test was used to compare irisin between different levels of body mass index (BMI). Spearman's correlation coefficient and partial correlation tests were applied to evaluate the correlation between nonparametric variables. The data were analyzed by SPSS version 16. In this study, $P$ value below 0.05 was respected as statistically significant for all tests.

\section{Results}

Table 1 illustrates some of the main characteristics of the subjects. The mean age was $61.25 \pm 11.7$ years. The majority of subjects were women (68\% of total), and duration of diabetes was 7.64 years.

The results of the correlational analysis are presented in Table 2 . In this study a positive correlation between serum irisin and age $(P=0.05, \mathrm{r}=0.19)$ and a negative correlation between irisin and BMI was detected $(P=0.01, \mathrm{r}=-0.25)$. Accordingly a positive correlation between age and serum $\mathrm{Cr}(P=0.02, \mathrm{r}=0.23)$ was detected.

Spearman's correlation analysis was performed to study the correlation between variables. The association between circulating irisin and biochemical and demographic parameters was shown in Tables 3 and 4. Data from both tables shows that, the majority of individuals had normal FBS (mg/dL), $\mathrm{HbA}_{1 \mathrm{C}}(\%)$, and GFR, however sUA (mg/ $\mathrm{dL})$ and $\mathrm{sCr}(\mathrm{mg} / \mathrm{dL})$ in the most of them were abnormal. There was a significant difference in serum irisin level between patients with normal and abnormal FBS (mg/ $\mathrm{dL}$ ), but there was no significant differences in irisin level between subjects with normal and abnormal other biochemical parameters. Also there was no significant difference between men and women and patients who taking insulin or oral hypoglycemic agents. 


\section{Discussion}

The present study was designed to determine relationship between serum irisin, glycemic indices, and renal function in diabetic patients. The results of this study indicated a positive correlation between irisin and age and a negative correlation between irisin and BMI. We also found a marginally negative correlation between irisin and FBS. However no significant correlation between circulating irisin and $\mathrm{HbA}_{1 \mathrm{c}}$ was detected. There was a significant difference in serum irisin level between the subject with normal and abnormal FBS, so it can be concluded that circulating irisin was higher in diabetic subjects with uncontrolled FBS. These results are consistent with other studies. Huh et al found circulating irisin concentrations were positively correlated with FBS (5) and Mehrabian et al determined serum irisin level correlated positively with insulin levels and FBS in normal weight obese subjects (10). Also Liu et al demonstrated the positive correlation between circulating irisin and FBS in nonobese, non-diabetic individuals. These results differ from some published studies (7). Zhang et al and Du et al in two different meta-analysis established that circulating irisin concentrations were significantly lower in patients with T2DM $(4,11)$. Sanchis-Gomar et al reported positive correlation between irisin level and $\mathrm{HbA}_{1 \mathrm{c}}$ in $\mathrm{T} 2 \mathrm{DM}$ patients with and without obesity (12). While, the majority of subjects were obese or over weight hence one explanation
Table 1. Basic characteristics of diabetic subjects $(n=103)$

\begin{tabular}{lcc}
\hline & Mean or median $^{c}$ & SD or range \\
\hline Age (years) & 61.25 & 11.70 \\
BMI (kg/m²) & 29.32 & 5.48 \\
Male (\%) & 32 & \\
Types of drug (\%) & & \\
Insulin & 10.9 & - \\
Oral hypoglycemic agents & 89.1 & - \\
Duration of diabetes (years) & 7.64 & 0.61 \\
FBS (mg/dL) & 140 & $109-183$ \\
HbA $(\%)$ & 7.50 & $6.10-9.00$ \\
sUA (mg/dL) & 4.70 & $3.95-5.60$ \\
Cr (mg/dL) & 0.90 & $0.80-1.00$ \\
eGFR (mL/min/1.73 m²) & 83.70 & $64.45-100.83$ \\
\hline GFR (MDRD) ${ }^{\text {b }}$ (cc/min) & 72.07 & $63.39-83.21$ \\
Irisin (ng/dL) & 2.60 & $1.80-3.40$ \\
\hline
\end{tabular}

Abbreviations: BMI, body mass index; $\mathrm{FBS}$, fasting blood sugar; $\mathrm{HbA}_{1 \mathrm{c}^{\prime}}$ glycosylated hemoglobin; sUA, serum uric acid; $\mathrm{Cr}$, creatinine.

${ }^{a}$ eGFR (estimated glomerular filtration rate) calculated from the results of blood creatinine, age, body size and gender.

${ }^{b}$ GFR (MDRD), calculated from the Modification of Diet in Renal Disease (MDRD) equation. Data for categorical variables are presented as percentages.

cParametric and non-parametric variables were extracted as mean \pm SD and median, quartile respectively.

Table 2. Correlation between serum irisin, glycemic indices, and renal function in diabetic patients

\begin{tabular}{|c|c|c|c|c|c|c|c|c|c|c|}
\hline & Irisin & Age & sUA & $\mathrm{sCr}$ & eGFR & GFR & FBS & $\mathrm{HbA}_{1 \mathrm{c}}$ & HTN & BMI \\
\hline Irisin & & $0.19^{a}$ & 0.01 & -0.01 & -0.14 & 0.01 & $-0.18^{b}$ & -0.09 & -0.07 & $-0.25^{a}$ \\
\hline Age & & & 0.1 & $0.23^{a}$ & $-0.66^{a}$ & $-0.4^{a}$ & -0.07 & -0.09 & -0.13 & -0.18 \\
\hline sUA & & & & $0.44^{\mathrm{a}}$ & -0.14 & $-0.37^{a}$ & -0.16 & $-0.27^{a}$ & 0.11 & $0.24^{\mathrm{a}}$ \\
\hline $\mathrm{Cr}$ & & & & & $-0.46^{a}$ & $-0.74^{\mathrm{a}}$ & -0.07 & -0.01 & -0.07 & 0.03 \\
\hline $\mathrm{GFR}\left(\mathrm{mL} / \mathrm{min} / 1.73 \mathrm{~m}^{2}\right)$ & & & & & & $0.72^{\mathrm{a}}$ & 0.05 & -0.08 & 0.19 & $0.54^{\mathrm{a}}$ \\
\hline GFR (MDRD) (cc/min) & & & & & & & 0.08 & 0.01 & $0.29^{\mathrm{a}}$ & 0.05 \\
\hline FBS & & & & & & & & $0.55^{\mathrm{a}}$ & 0.16 & -0.1 \\
\hline $\mathrm{HbA}_{1 \mathrm{c}}$ & & & & & & & & & 0.16 & -0.18 \\
\hline
\end{tabular}

Abbreviations: $\mathrm{BMI}$, body mass index; $\mathrm{FBS}$, fasting blood sugar; $\mathrm{HbA}_{1 c^{\prime}}$ glycosylated hemoglobin; sUA, serum uric acid; sCr, serum creatinine. eGFR, estimated glomerular filtration rate; HTN, hypertension; MDRD, Modification of Diet in Renal Disease.

a $\mathrm{P}<0.05$ is significant; ${ }^{\mathrm{b}}$ is marginally significant.

Table 3. Compare circulating irisin between diabetic subjects with normal and abnormal biochemical characteristics

\begin{tabular}{|c|c|c|c|c|c|c|c|}
\hline & \multicolumn{3}{|c|}{ Normal } & \multicolumn{3}{|c|}{ Abnormal } & \multirow{2}{*}{$P$ value } \\
\hline & Percent & Mean (ng/dL) & SD & Percent & Mean (ng/dL) & SD & \\
\hline sUA (mg/dL) & 85.1 & 2.98 & 0.22 & 14.9 & 2.86 & 0.21 & 0.71 \\
\hline $\mathrm{Cr}(\mathrm{mg} / \mathrm{dL})$ & 95 & 2.86 & 0.15 & 5 & 3.2 & 0.49 & 0.37 \\
\hline eGFR $\left(\mathrm{mL} / \mathrm{min} / 1.73 \mathrm{~m}^{2}\right)$ & 39.6 & 2.67 & 1.18 & 60.4 & 3.10 & 1.81 & 0.29 \\
\hline GFR (MDRD) & 10.9 & 2.88 & 1.84 & 89.1 & 2.94 & 1.58 & 0.37 \\
\hline $\mathrm{FBS}(\mathrm{mg} / \mathrm{dL})$ & 89.1 & 2.81 & 1.55 & 10.9 & 3.92 & 1.73 & 0.009 \\
\hline $\mathrm{HbA}_{1 \mathrm{c}}(\%)$ & 43.6 & 2.87 & 1.37 & 56.4 & 2.98 & 1.77 & 0.84 \\
\hline
\end{tabular}

Abbreviations: FBS, fasting blood sugar; $\mathrm{HbA}_{1 c^{\prime}}$ glycosylated hemoglobin; sUA, serum uric acid; Cr, creatinine; eGFR, estimated glomerular filtration rate; MDRD, Modification of Diet in Renal Disease. 
Table 4. Association of circulating irisin with demographic parameters and BMI levels in diabetic subjects

\begin{tabular}{lccc}
\hline & Mean (ng/dL) & SD & $P$ value \\
\hline Sex & & & 0.58 \\
Female & 2.82 & 0.16 & \\
Male & 3.18 & 0.35 & \\
Age & & & 0.52 \\
<45 & 2.42 & 0.83 & \\
$>45$ & 2.97 & 1.64 & \\
BMI (kg/m $\left.{ }^{2}\right)$ & & & $0.05^{\mathrm{a}}$ \\
$\leq 18.5$ & 6.23 & 4.2 & \\
$18.5-24.9$ & 3.22 & 1.45 & \\
$25-24.9$ & 3.03 & 1.52 & \\
$>30$ & 2.49 & 0.96 & \\
Types of drug & & & 0.48 \\
Insulin & 2.95 & 1.58 & \\
OHA & 2.8 & 1.87 & \\
\hline
\end{tabular}

Abbreviations: BMI, body mass index; $\mathrm{OHA}$, oral hypoglycemic agents. a $P<0.5$ is significant.

for our result is "irisin resistance" in obese people as same as leptin and insulin resistance (13). Our findings revealed that no correlation between irisin and renal function tests and indices such as sUA, Cr, and GFR was detected. Also no significant difference between the subjects with normal and abnormal renal function markers was seen. The findings of the current study do not support the previous research $(6,7)$, while, Wen et al established that plasma irisin levels were significantly decreased in chronic kidney disease (CKD) patients (6,7). Accordingly, Liu et al found that circulating irisin was significantly decreased in diabetic patients with renal insufficiency compared to T2DM patients with normal renal function. They found that circulating irisin was independently associated with GFR (7). Yang et al determined that high serum irisin level was associated with reduced risk of chronic kidney disease (CKD) (14). A possible explanation for this inconsistency may be due to studied different populations.

\section{Conclusion}

This study presented the relationship between serum irisin, glycemic indices, and renal function in diabetic subjects. This research has shown a positive correlation between irisin and age and a negative correlation between irisin and BMI $(P=0.01, r=-0.25)$. We also showed a significant difference in serum irisin level between the subjects with normal and abnormal FBS. In general, it seems that serum irisin concentration was increased with age and decreased with BMI and it was higher in subject with abnormal FBS. However, further research is suggested to provide inclusive understanding of irisin associated physiological effects and possible implications in clinical conditions.

Limitations of the study

Low proportion of patients is a limitation of our study.

\section{Authors' contribution}

All authors participated to design of the study. LM managed the research. MS performed the investigation. AA and MK analyzed the data. MK prepared the manuscript. All authors read, revised and approved the manuscript.

\section{Conflicts of interest}

The authors declare that they have no competing interests.

\section{Ethical considerations}

Ethical issues (including plagiarism, data fabrication, double publication) have been completely observed by the authors.

\section{Funding/ Support}

This study was extracted from MD thesis of Maryam Sadoughi (\# 1252) and supported by the Vice-Chancellery for Research and Technology of Shahrekord University of Medical Sciences.

\section{References}

1. Guariguata L, Whiting D, Hambleton I, Beagley J, Linnenkamp U, Shaw J. Global estimates of diabetes prevalence for 2013 and projections for 2035. Diabetes Res Clin Pract. 2014;103:137-49. doi: 10.1016/j. diabres.2013.11.002.

2. Boström P, Wu J, Jedrychowski MP, Korde A, Ye L, Lo JC, et al. A PGC1-alpha-dependent myokine that drives brown-fat-like development of white fat and thermogenesis. Nature. 2012;481:463-8. doi: 10.1038/ nature 10777.

3. Crujeiras AB, Pardo M, Arturo RR, Santiago NC, Zulet M, Martínez JA, et al. Longitudinal variation of circulating irisin after an energy restriction-induced weight loss and following weight regain in obese men and women. Am J Hum Biol. 2014;26:198-207. doi: 10.1002/ajhb.22493.

4. Du XL, Jiang WX, Lv ZT. Lower circulating irisin level in patients with diabetes mellitus: a systematic review and meta-analysis. Horm Metab Res. 2016;48:644652. doi: 10.1055/s-0042-108730

5. Huh JH, Ahn SV, Choi JH, Koh SB, Chung CH. High Serum irisin level as an independent predictor of diabetes mellitus: a longitudinal populationbased study. Medicine. 2016;95:e3742. doi: 10.1097/ MD.0000000000003742.

6. Wen MS, Wang CY, Lin SL, Hung KC. Decrease in irisin in patients with chronic kidney disease. PLoS One. 2013;8:e64025. doi: 10.1371/journal. pone.0064025.

7. Liu JJ, Wong MD, Toy WC, Tan CS, Liu S, Ng XW, et al. Lower circulating irisin is associated with type 2 diabetes mellitus. J Diabetes Complications. 2013;27:365-9. doi: 10.1016/j.jdiacomp.2013.03.002.

8. Zhang HJ, Zhang XF, Ma ZM, Pan LL, Chen $\mathrm{Z}$, Han $\mathrm{HW}$, et al. Irisin is inversely associated with intrahepatic triglyceride contents in obese 
adults. J Hepatol. 2013;59:557-62. doi: 10.1016/j. jhep.2013.04.030.

9. Hojlund K, Boström P. Irisin in obesity and type 2 diabetes. J Diabetes Complications. 2013;27:303-4. doi: $\quad 10.1016 /$ j.jdiacomp.2013.04.002.

10. Mehrabian S, Taheri E, Karkhaneh M, Qorbani M, Hosseini S. Association of circulating irisin levels with normal weight obesity, glycemic and lipid profile. J Diabetes Metab Disord. 2015;15:17. doi: 10.1186/ s40200-016-0239-5.

11. Zhang C, Ding Z, Lv G, Li J, Zhou P, Zhang J. Lower irisin level in patients with type 2 diabetes mellitus: A case-control study and meta-analysis. J Ddiabetes. 2016;8:56-62. doi: 10.1111/1753-0407.12256.
12. Sanchis-Gomar F, Lippi G, Mayero S, Perez-Quilis C, Garcia-Gimenez JL. Irisin: a new potential hormonal target for the treatment of obesity and type 2 diabetes. J Diabetes. 2012;4:196. doi: 10.1111/j.17530407.2012.00194.x.

13. Choi YK, Kim MK, Bae KH, Seo HA, Jeong JY, Lee WK, et al. Serum irisin levels in new-onset type 2 diabetes. Diabetes Res Clin Pract. 2013;100:96-101. doi: 10.1016/j.diabres.2013.01.007.

14. Yang S, Xiao F, Pan L, Zhang H, Ma Z, Liu S, et al. Association of serum irisin and body composition with chronic kidney disease in obese Chinese adults: a cross-sectional study. BMC Nephrol. 2015;16:16. doi: 10.1186/s12882-015-0009-5.

Copyright ( 2017 The Author(s); Published by Nickan Research Institute. This is an open-access article distributed under the terms of the Creative Commons Attribution License (http://creativecommons.org/licenses/by/4.0), which permits unrestricted use, distribution, and reproduction in any medium, provided the original work is properly cited. 